\title{
ANÁLISIS DEL DIÁLOGO INTERCULTURAL A PARTIR DEL MODELO PEDAGÓGICO
}

\author{
Teresa Tonantzin ORTIZ RODRÍGUEZ \\ Universidad Intercultural del Estado de Puebla (México) \\ tonantzin.ortiz@uiep.edu.mx
}

\section{ANALYSIS OF INTERCULTURAL DIALOGUE FROM PEDAGOGIC MODEL}

Resumen: Desde finales del siglo pasado hasta lo que va del siglo XXI, la diversidad cultural ha sido admitida como una riqueza para el desenvolvimiento de la sociedad; sin embargo, los modelos de desarrollo, sus políticas educativas para concretarlos y sus modelos pedagógicos poco reflejan en los hechos la contundencia de la diversidad cultural como detonador de procesos de desarrollo en equidad, para eliminar posiciones racistas, discriminatorias y de explotación de grupos hegemónicos a grupos subordinados. El movimiento indígena en México originado desde los primeros años de la colonia española persistente hasta la actualidad, ha abierto espacios ante la hegemonía nacional como lo son: las escuelas públicas de nivel básico, medio superior y superior. En donde lo indígena y los indígenas han dejado de ser los beneficiaros de políticas educativas integracionistas conducentes a eliminar paulatinamente su cultura para ser parte de la cultura nacional; ahora son vistos como agentes activos para la toma de decisiones en su propio proceso educativo, para ello, se ha propuesto el llamado modelo pedagógico intercultural, este artículo debate sobre cómo se ha llevado a cabo dicho modelo en un contexto de nivel medio superior, es decir, en la Universidad Intercultural del Estado de Puebla.

Abstract: Since late the century passed so that you go from XXI the century XXI, the cultural diversity has been allowed like a riches for the development of the society; However, the models of development, his educational policies to concretize them and his pedagogic models little reflect in the facts the force of the cultural diversity like detonator of processes of development in equity, to eliminate racist, discriminatory and exploitation positions of hegemonic groups to subordinate groups. The indigenous movement in Mexico originated from the tender age of the Spanish persistent cologne to the present time, you have opened spaces in front of the national hegemony as the public schools of basic level are it, I mediate superior and superior. Where they have stopped being the indigenous and the indigenous to benefit to them of educational policies conducive integrationists to eliminate his culture to be a part of the national culture step by step; Now they are obvious like active agents for the overtaking in his own educational process, for it, debate has proposed the so-called pedagogic intercultural model, this article itself be more than enough how the aforementioned model in a context of half superior level has taken effect, that is, at the Intercultural University of Puebla's State.

Palabras clave: Educación intercultural. Metodología de la enseñanza-aprendizaje. Educación superior. Vinculación comunitaria. Indígenas

Intercultural education. Methodology of teaching learning. Higher education. Communal linkage. Natives 


\section{Introducción}

Recurrir al tema de la interculturalidad como punto de partida para un debate teórico-metodológico conlleva muchas aristas, considero pertinente contextualizarlo para argumentar su factibilidad como paradigma interpretativo y comprensivo de la realidad mexicana ante la convergencia de diversas culturas en un mismo territorio tanto físico como simbólico.

Un ámbito de nuestra pertinencia para comenzar este debate teórico-metodológico es precisamente la educación superior, si nos consideramos docentes comprometidos con nuestro quehacer cotidiano; hacerlo, significa entrar de lleno a reflexionar si el tan mencionado diálogo de saberes propios en un espacio educativo como la universidad es una realidad contundente.

Si bien es cierto, la interculturalidad como paradigma educativo ha venido sustentando políticas públicas y prácticas pedagógicas durante los últimos diez años, éstas se han centrado a niveles básicos, preferentemente de educación primaria. Por consiguiente, el objetivo principal de este artículo es analizar los alcances pedagógicos del paradigma de la interculturalidad dentro de la educación superior para el desarrollo de competencias a profesionistas del ámbito rural e indígena, mediante una visión crítica realizada a la vinculación comunitaria como metodología de enseñanza-aprendizaje coherente al modelo pedagógico de la Universidad Intercultural del Estado de Puebla, la cual conlleva a hacer realidad un diálogo entre "iguales".

\section{Pluriculturalidad, multiculturalidad o interculturalidad: la encrucijada pedagógica}

La historia de la humanidad está marcada de encuentros y desencuentros producto de migraciones para la búsqueda de mejores condiciones de vida, guerras de conquista y transacciones comerciales. La movilidad de los grupos sociales con respecto a sus espacios físicos ha provocado a través de dicha historia un gran dinamismo en la cultura, es decir, la cultura no es una pieza de museo únicamente; principalmente, es el fundamento del ser humano para concebirse como tal y reproducirse, por consiguiente, los encuentros y desencuentros históricos han repercutido notablemente en la construcción y reconstrucción de las distintas culturas de los pueblos, el análisis del contacto entre sociedades distintas permite entender los procesos de adquisición, utilización y transformación del conocimiento para la conformación del espacio social dentro del cual se dan cita las diferentes perspectivas de los sujetos y actores sociales participantes (Landázuri, 2002).

México es un claro ejemplo de la convergencia de culturas desde las migraciones de los grupos nómadas de la prehistoria hasta el compacto, pero bien diversificado, territorio denominado Mesoamérica. Y qué decir, sobre la guerra de conquista y colonialismo, la cual introdujo dos raíces más, a la ya diferenciada conformación cultural mesoamericana: la europea y la africana. Todo este transcurrir histórico, como es bien reconocido por nosotros, llamó la atención de los antropólogos por su función como intermediarios intelectuales de la hegemonía política, y por tanto, educativa y productiva.

La diversidad cultural mexicana, se convirtió en el objeto de estudio preferente de la antropología, pero también de otras áreas de conocimiento tales como la sociología y la historia, porque aceptaron a la diversidad como un tema central para la comprensión de una realidad como la nuestra.

Un primer intento para la comprensión de la diversidad étnica construida históricamente por los distintos sujetos sociales de la compleja sociedad mexicana, fue mirarla como un collage o mosaico; el primer término para denominar dicho mosaico, desde la visión académica, fue la pluriculturalidad. Sin embargo, éste término se centra únicamente en confirmar la existencia de una diversidad cultural yuxtapuesta, en donde los distintos grupos étnicos, quienes comparten un mismo territorio físico, no se identifican dentro del mismo territorio simbólico; en este sentido, sus distintas intersubjetividades para la aprehensión y despla- 
zamiento dentro de dicho territorio, no están interconectadas, por consiguiente, las etnias contenidas en ésta, únicamente se conciben como sobrepuestas sin una vinculación directa; en donde la diversidad cultural se ve como un obstáculo para el desarrollo.

No obstante lo anterior, la postura de la pluriculturalidad permite evidenciar las relaciones de poder y correlación de fuerza, pero sin niveles de negociación entre ellas. Los distintos grupos diferenciados culturalmente mantienen una relación de dominación, y por ende, en continuo conflicto sin llegar a niveles de negociación, en este enfoque finalmente, aunque se reconoce la diversidad, los grupos que las representan nunca llegan a ponerse de acuerdo para poder convivir.

Otro enfoque para la comprensión de la diversidad cultural y cómo abordarla en políticas públicas es el de la multiculturalidad, en este se reconoce la interrelación mantenida entre las distintas etnias dentro de un territorio físico-simbólico, pero justamente éste se encuentra en constante disputa. Es decir, las relaciones hegemónicas, en donde las etnias negocian sus territorios simbólicos dentro del mismo territorio físico para la aceptación de una cultura nacional como hegemónica y culturas subordinadas, dentro de las cuales se encuentran las populares y las indígenas. Este enfoque, también ha sustentado políticas públicas tendientes a la eliminación de la diferencia, por concebirla como un obstáculo para el desarrollo de la nación o de la sociedad.

El Movimiento Zapatista de Liberación Nacional acaecido en 1994 evidenció la postura del enfoque multicultural; cuando las voces indígenas irrumpieron hacia nuevos niveles de negociación. Porque su cultura subordinada desde la conquista española bajo el signo de un indigenismo tendiente al etnocidio disfrazado, ya sea primero, con una política segregacionista durante la colonia; posteriormente con una política incorporativista en la independencia; y finalmente, con una política integracionista durante la época pos revolucionaria, se mantuvo calladamente, y cuando quiso alzar la voz fue brutalmente violentada (Díaz-Polanco, 1987).

La sobrevivencia de la cultura indígena durante tanto tiempo, se debió a su forma especial de negociar sus espacios físicos y simbólicos para poder reproducirse, la cual funcionó eficientemente durante siglos: la rebeldía del subordinado expresada en un lenguaje oculto, la llamada resistencia pasiva; le permitió persistir durante más de quinientos años dentro de los territorios definidos y asignados por los hegemónicos, e incluso, abarcar los espacios simbólicos de la cultura nacional, insertándose en la médula cotidiana de cada uno de los hogares de esos grupos dominantes mediante su gastronomía, la música, el uso de vocablos de lenguas indígenas, la religiosidad popular, entre otros aspectos (Bonfil Batalla, 1990).

La resistencia pasiva indígena se mostró y ha mostrado como una potente estrategia de adaptación y adopción de la cultura hegemónica, gracias a lo cual ha trascendido en el tiempo; de tal manera, los encuentros y desencuentros multiculturales, han sido manejados por los indígenas como un diálogo permanente desde el lenguaje del oprimido.

En el lenguaje del oprimido (Scott, 2000) se escucha atentamente al hegemónico, en donde el subordinado retoma de sus propuestas y de su discurso, sólo aquello conveniente para su propia sobrevivencia; esa sutil afirmación a todo, pero sin llegar al detalle de cuándo y cómo (Landázuri, 2002).

Más allá de las necesarias rebeliones indígenas ocurridas durante toda la historia de la conformación de la nación mexicana, la resistencia pasiva ha garantizado los senderos de la sobrevivencia india como la denomina Bonfil Batalla (1990). Entonces, ¿por qué seguimos sin admitir, cómo la negociación indígena desde siempre, ha mantenido un diálogo constante con las distintas etnias dentro del territorio nacional, cuyo producto ha sido un intercambio cultural y no un choque brutal constante, una pérdida, una ruptura sin posibilidad de reestablecerse?

La sustitución y la incorporación de ciertos elementos culturales hegemónicos aceptados por los indígenas dentro de su cotidianidad, representan el intercambio producto del 
diálogo, el cual, en ocasiones, ha llegado a niveles de discusión violenta, al romperse el diálogo explicito u oculto entre los distintos grupos. Sin embargo, el diálogo del oprimido ha persistido como garante de su cultura, por ello, no podemos negar relaciones interétnicas, intralingüísticas e interreligiosas durante el desarrollo histórico de nuestro país; pero en el plano normativo el lenguaje del oprimido adquiere otros matices bajo principios de equidad, de diferencia y de interacción positiva.

El diálogo indígena mantenido con los hegemónicos durante cientos de años, ha sido vertical, no entre iguales, marcados por situaciones en disputa, como bien lo establece el enfoque multicultural.

Los niveles de negociación expresado en este artículo como un diálogo vertical entre oprimidos y hegemónicos, es sumamente visible en el contexto en el cual se encuentra inserta la Universidad Intercultural del Estado de Puebla, cuya matrícula se compone principalmente de jóvenes originarios de poblaciones indígenas de la Sierra Nororiental de Puebla; región ubicada en el centro del territorio mexicano.

La Sierra Nororiental se convirtió durante la colonia española en zonas de refugio de indígena totonacos y nahuas, quienes decidieron habitar la serranía ante la política segregacionista de ésta época. Los pueblos originarios pudieron conservar muchas formas culturales y actividades económicas de la época prehispánica, a pesar de haber sido evangelizados por misiones de frailes franciscanos.

De clima semicálido a cálido húmedo con lluvias todo el año, la región serrana permite el desarrollo de bosques mesófilos de montaña y selva tropical, en donde se distinguen los helechos arborescentes, como testigos de una época prehistórica. La orografía cuenta con una gran cantidad de grutas, cascadas, pozas de agua, formaciones rocosas y manantiales; sin embargo, lo más sobresaliente en términos del patrimonio es la herencia prehispánica del lugar: habitada por poblaciones de origen náhuatl y totonaco como grupo mayoritario.

Desde la conquista, los indígenas fueron víctimas de la explotación y discriminación por parte de los grupos dominantes, como otras etnias, la población indígena vivió una gran segregación no sólo cultural, económica y política, sino también espacial al huir del mestizaje, se refugiaron en las tierras agrestes de la serranía de poco interés económico para los colonizadores, esto les permitió conservar su estilo de vida. Fue a partir de las Leyes de Reforma a finales en el siglo XIX, cuando comienza la adjudicación de tierras y la introducción a la zona de población mestiza, quienes fortalecieron el cultivo de café y la producción de aguardiente (a base de caña), debido a la influencia de la introducción del cultivo de café en 1870 en Córdoba, Veracruz (Hernández-Loeza, 2011), Así mismo la ganadería extensiva vacuna para engorada; a partir de este repoblamiento, la zona sufrió de la apropiación de tierras mediante la usura y el endeudamiento de los indígenas por la compra de aguardiente.

El intermediarismo y caciquismo mediante el compadrazgo fue la práctica común para enganchar a dicha población, lo cual los colocó en una situación de alta marginalidad económica y social durante finales del s XIX hasta los años setenta del siglo pasado. En la actualidad, con la caída del precio de café, ahora la pimienta lo ha sustituido, y todavía en la cabecera municipal de Huehuetla municipio al cual pertenece Lipuntahuaca, localidad en donde fue construida la Universidad; podemos observar cómo desde el amanecer llegan los indígenas cargando en sus espaldas o en bestias de carga, los bultos de café y pimienta para entregárselos a los intermediarios, quienes se los pagan a precios inferiores del establecido en el mercado.

Generalmente, el intermediario es dueño de la tienda de abarrotes y bienes de consumo domésticos, los cuales no son producidos por los indígenas en sus pequeñas parcelas; de tal manera, durante el año, ante la falta de dinero, suelen pedir dichos productos industrializados a crédito, así cuando es la cosecha del café y pimienta, éstas ya están comprometidas con los tenderos para pagar la deuda. O bien, todavía en los años ochenta del siglo pasado, los dueños de las destiladoras de aguardiente se lo vendían a crédito a los indígenas pro- 
vocando fuerte problemas de alcoholismo en esta población; quienes, a falta de dinero se vieron obligados a liquidar su cuenta al ceder sus terreros a los dueños de las destiladora.

Como podemos observar, en esta región, los indígenas han estado en negociación con los grupos hegemónicos, se ha dado un diálogo vertical, el cual ha conllevado a una discriminación brutal dirigida hacia la población indígena de la región serrana, y por tanto, las generaciones de jóvenes a partir del siglo pasado se encontraron en una tensión constante entre conservar su cultura o insertarse a la cultura nacional para dejar de ser los explotados. La pérdida cultural o el choque violento; es innegable en la realidad indígena, pero los niveles de negociación, ya descritos, permiten admitir la interculturalidad llevada a cabo por este grupo étnico desde los encuentros y desencuentros intencionalmente provocados por este grupo al emigrar a otros lugares, o violentados por la incursión de otros grupos como la invasión mexica a la región totonaca, la guerra de conquista española, o de la población mestiza durante el siglo XIX en la Sierra.

En concreto, la interculturalidad es un fenómeno continuo en la historia de los pueblos indígenas desde la época prehispánica (la diversidad cultural de Mesoamérica también era vasta, pero la interconexión simbólica territorial la definía como un conjunto compacto), durante la colonia, la independencia, la pos revolución y hasta nuestros días. ¿Por qué entonces ahora surge una nueva categoría de análisis en el ámbito académico denominada interculturalidad?

Porque si bien en los hechos la interculturalidad es una realidad en México desde su gestación como nación, el diálogo en el cual se manifiesta, como ya dije, es vertical, en condiciones reales de desventaja para el indígena; por lo tanto, las voces apagadas en años anteriores buscan nuevas resonancias ante un movimiento global de dignificación de los derechos humanos.

La interculturalidad entonces se está construyendo como un paradigma más abarcativo de una realidad en donde, las relaciones emanadas del diálogo, transfieren desde un punto de partida a un punto de llegada, en condiciones horizontales, en un plano de igualdad, lo cual conlleva a un proceso de selección, transporte y selección de lo transmitido y admitido en esa transferencia de mensajes. (Dietz y Mateos Cortés, 2011: 39) Por consiguiente, la interculturalidad implica un intercambio asociado durante el encuentro de individuos o grupos socialmente distintos debido a su identidad cultural. Algunos autores, por dicha característica de transferencia, ubican a la interculturalidad como un diálogo entre culturas basado en el respeto y aceptación a la diferencia.

La interculturalidad, si bien ha reflejado históricamente un diálogo vertical entre dominantes y dominados, en la actualidad busca establecer las bases para eliminarlo, pero para lograrlo se requieren procesos educativos incidentes en las formas de pensamiento colonizado y colonizador arraigadas en los pueblos indígenas y no indígenas de nuestro país. En este sentido, ¿cómo emplear a la interculturalidad como el sustento de un modelo educativo alternativo al ya existente en México?

\section{De la educación indígena a la educación intercultural}

La educación indígena en México ha tenido graves problemas de aplicación porque en la disputa por el territorio simbólico, ha conducido al paulatino etnocidio al imponer conocimientos de la cultura nacional ajenos a la cultura de los pueblos indígenas. Fuertes críticas se han suscitado en torno a esta política, la principal es proponer un bilingüismo bajo el pretexto de la conservación cultural, en donde la lengua materna sólo se usa como vehículo para traducir la cultura nacional y de esta forma, sea mejor entendida y aceptada por la población indígena a edades tempranas.

La educación indígena fue propuesta nuevamente desde un diálogo vertical, en donde lo hegemónico escuchó las voces indígenas, pero bajo la conveniencia del proyecto de desa- 
rrollo nacional, en donde la cultura indígena se valoraba como un obstáculo ante el progreso tecnológico. La incorporación e integración de lo indígena sólo era posible mediante la transformación del pensamiento retrógrada de éste. Este tipo de educación fue el arma más eficaz empleada por la política indigenista para hacer partícipes a las diversas etnias del país a la vida nacional, se transformaron en objeto de beneficio, porque la política educativa fue diseñada desde la concepción del otro y no desde ellos mismos.

En la educación indígena, el modelo de desarrollo económico y cultural mexicano, los indios si tenían cabida, pero el precio a pagar para lograrlo era ser transformados; es decir, la pérdida de su esencia: la desindianización.

Dicho lo anterior, al retomar el enfoque intercultural en educación se ha derivado en varios objetivos: para conservar, para prevenir el racismo, para transformar relaciones de poder, para interactuar bajo los principios de respeto y equidad; y finalmente para descolonizar (Dietz y Mateos Cortés, 2011). Cada uno de estos objetivos han sido desarrollados de manera aislada, el reto es cumplir todos ellos sin caer en existencialismos o radicalismos.

La transformación de las relaciones de poder desde una interacción basada en el respeto y la equidad, conllevan a la descolonización del pensamiento; por lo tanto, toda educación intercultural debe buscar tales objetivos. ¿Cómo lograrlo? Mediante la recuperación de los imaginarios sociales coadyuvantes a interactuar bajo dichos principios, los cuales rescatarán las necesidades sentidas de la población indígena en su conjunto, no sólo de los y las estudiantes.

El modelo pedagógico intercultural conlleva al desarrollo de competencias de corte aptitudinal, actitudinal, de valores y normas; de esta forma, ofrece una formación integral a los y las estudiantes basados en el principio de respeto y equidad, en donde toda cosmovisión es libre de ser expresada para mantener un diálogo e intercambio de saberes horizontal, y no se pretende el predominio de una sobre otra; más bien dicho intercambio es tendiente a la erradicación paulatina de las relaciones hegemónicas.

Como he tratado de dejar en claro en la política indigenista en donde ha predominado el enfoque pluricultural y multicultural, las relaciones hegemónicas persisten e incluso se han fortalecido al cosificar los saberes tradicionales como parte de la cultura hegemónica desde los mestizos para los indígenas; de tal manera, se han justificado políticamente las estrategias asistencialistas, en donde, las etnias son tratadas como objetos de beneficio y no sujetos.

En la Sierra Nororiental, la educación indígena tuvo ese tinte incorporativo e integracionista durante la época independiente y pos-revolucionaria; no es sino hasta la última década del siglo XX, cuando irrumpe a México el modelo pedagógico intercultural y a esta región, ya iniciado el presente siglo.

En las universidades interculturales, bajo su modelo pedagógico, no sólo se busca un aprendizaje significativo en el sentido estricto desarrollado teóricamente por Ausbel (1983) bajo el paradigma del construccionismo; sino fundamentalmente, transformar la educación en verdaderos estilos de vida, y por ende, proyectos de vida individual y social.

\section{La vinculación comunitaria como estrategia pedagógica para un diálogo horizontal}

La transformación del sistema educativo desde la interculturalidad se vuelve una realidad mediante el empleo de una metodología de enseñanza-aprendizaje coherente con dicho modelo. Desde la interculturalidad, se ha propuesto a la vinculación comunitaria para tal fin.

Estamos retomando como metodología de enseñanza-aprendizaje a la propuesta didáctica diseñada para atender las necesidades de formación profesional de las y los discentes; es decir, todo el conjunto de elementos pedagógicos coadyuvantes a facilitar el PEA, por tanto, la metodología determina el cómo llevar a cabo el proceso formativo. Los componentes pedagógicos incluyen: el objetivo, el contenido, el método, los medios, las formas 
organizativas de la enseñanza y la evaluación, estos se relacionan entre sí y persiguen un fin común (Rico, 2004 y Rivera, 2003).

Los componentes de una metodología de enseñanza-aprendizaje son:

"Objetivo: Es la categoría rectora, ésta expresa las aspiraciones educativas del mismo, en función del desarrollo de la personalidad de los estudiantes, y por consiguiente, la transformación a lograr en el estudiante. Contenido: Es la parte de la cultura de la humanidad (conocimientos científicos y tradicionales) expresada en una asignatura. Estrategias didácticas: los modos de organizar la actividad cognoscitiva de los estudiantes para asegurar el dominio de los conocimientos; es decir, las dinámicas grupales sustentadas por un paradigma educativo; y las técnicas grupales, entendidas como las actividades de enseñanza-aprendizaje. Medio: Es el soporte material de la estrategia didáctica, sirven al profesor para objetivizar la enseñanza y evitar el verbalismo, existen una gran variedad de ellas, desde el más antiguo (pizarrón y gis) hasta los más modernos (tabletas, pizarrones interactivos, plataformas). Formas organizativa de la enseñanza: Se compone de elementos, los cuales hacen referencia al sistema educativo expresado en los lineamientos de planes y programas institucionales contenidos en la currículo oficial. Evaluación: Es la comprobación del grado de cumplimiento de los objetivos y los resultados obtenidos, en términos de la calidad educativa” (Rico, 2004 y Rivera, 2003).

Si retomamos como ejes rectores de una metodología de enseñanza-aprendizaje cada uno de los componentes descritos en párrafos anteriores, es una obligación preguntarnos si la vinculación cumple con éstos como para ser considerada una metodología.

El principal objetivo de la vinculación comunitaria o con la comunidad es lograr un desarrollo autónomo porque se basa en el paradigma del constructivismo sociocultural, es decir, la interiorización de los signos sociales de la internalización de la cultura y las relaciones sociales; de tal forma, la vinculación es una práctica constructiva, consciente, intencional y activa (Muñoz y Santini, 2009: 159-160).

La vinculación parte de un diálogo de saberes en forma elíptica porque se genera desde y para los distintos actores educativos participantes. La vinculación comunitaria define a dichos sujetos sociales a partir de sus propias necesidades educativas, al considerar no sólo al estudiante, al profesor o a las autoridades educativas universitarias como protagonistas del escenario del aprendizaje y enseñanza; sino también considera las expectativas de los integrantes de un pueblo: estudiantes, profesores de otros niveles educativos, miembros de algunas asociación civil, empresa o cooperativa, autoridades locales, amas de casa, campesinos, artesanos, entre otros. Porque prevé la planeación, organización, operación y evaluación en cuanto a la atención de problemáticas y necesidades específicas mediante la articulación de la investigación con la docencia (Muñoz y Santini, 2009: 157).

La vinculación con la comunidad es un ejercicio constante semestral, no es un servicio a la comunidad o un servicio social, el fundamento didáctico es el aprendizaje basado en proyectos reales propuestos a partir de un diagnóstico hecho por profesores y estudiantes. Las necesidades detectadas son teorizadas desde la academia para darles una respuesta o satisfactor, cuya viabilidad es factible a corto, mediano y largo plazo. En este tipo de propuesta pedagógica el estudiante y el docente no logran obtener resultados y cumplir objetivos sin la participación de los representantes comunitarios.

Dicho de otro modo, para que los y las estudiantes puedan realizar su vinculación cada semestre, debe antecederles el diseño de un proyecto de gestión comunitaria, en donde ellos tienen un papel protagónico. Los medios de enseñanza-aprendizaje dentro de la vinculación son tan variados como diversos los contextos en donde se lleva a cabo: en el aula, 
el pintarrón, los marcadores, los proyectores, las computadores; en campo, la oralidad, las herramientas de trabajo agrícola, las técnicas de laboratorio y su equipo, el telar de cintura, la cocina al fogón, entre otros.

El modelo pedagógico intercultural junto con la vinculación comunitaria, tienen un reconocimiento oficial y forman parte de una política nacional educativa, están regidas por el sistema mexicano, como una forma de organización externa al aula y al campo en donde se dan cita los diálogos de saberes entre estudiantes, docentes y gente de la comunidad (Muñoz y Santini, 2009).

Como vemos la vinculación si es una metodología porque cuenta con todos los componentes de la misma, pero para el caso de la evaluación aún faltan lineamientos por llevar en práctica y reajustar. Cuando menos para el caso de la UIEP, no se ha sistematizado todas las experiencias de vinculación en años anteriores, las cuales nos permitan perfilar indicadores precisos para ver sus alcances y limitantes.

\section{Consideraciones finales}

La interculturalidad acotada desde un modelo pedagógico con su particular metodología de enseñanza-aprendizaje denominada vinculación comunitaria, es una realidad objetiva en la UIEP, pero la descolonización de pensamientos tanto de los y las estudiantes, e incluso de los docentes y autoridades educativas, como de los habitantes de las localidades indígenas como mestizas llevará todavía un proceso más arduo y paulatino.

El diálogo intercultural sigue siendo fundamentalmente vertical, nuestras y nuestros estudiantes cuando son confrontados con las teorías y técnicas científicas sufren metamorfosis camaleónicas y dejan de ser indígenas para ser "científicos" y "gente de razón"; y suelen referirse de sus coterráneos, cuando plantean un proyecto o exponen sobre alguna investigación producto de trabajo en campo en sus propias comunidades, o bien, citan a autores estudiosos del asunto indígena; como "los otros", "los indígenas" o "los de los pueblos originarios". Y cuando están frente a ellos, vuelven a ser parte de éstos y se identifican como tal.

El riesgo de una interculturalidad vertical, es el ejemplo de toda nuestra historia mexicana, relaciones hegemónicas disfrazadas con distintas máscaras como la democracia, la igualdad, la tolerancia, entre otras; cuando los rostros genuinos reflejarían, respeto, equidad, empatía. Porque, lamentablemente un indio reidianizado desde la academia, desde la escuela; si no es bien conducido pedagógicamente hablando en términos de conciencia social, puede caer en exitencialismos radicales: de pasar de ser víctima del racismo a ser racista, de haber sido objeto de imposiciones, a ser sujeto impositor, de haber sido víctima de la intolerancia a ser intolerante.

Debemos como docentes reflexionar ampliamente si los resultados de la vinculación y nuestro quehacer cotidiano dentro del aula, no están conduciendo a una reidianización exacerbada e idiologizada en sentido contrario; lo cual conduce, inevitablemente también a un diálogo vertical, entonces preguntémonos antes de cualquier acción pedagógica primero: ¿con esta acción logro realmente un diálogo horizontal?

\section{Bibliografía}

Araya, valeria et.al (2007) construccionismo: orígenes y perspectivas, LAURUS, VOL. 4, NÚM. 24, MAYOAGOSTO, PP. 76-92. DISPONIBLE EN HTTP/WWW.REDALYC.ORG/ARTICULO.OA?ID=76111485004.

Ausubel, novak_hanesian (1983) psicología educativa. un punto de vista cognitivo, $2^{\mathrm{a}}$ Ed., Trillas, México.

Baeza, Manuel Antonio (2003) Imaginarios sociales. Apuntes para la discusión teórica y metodológica, Editorial Universidad de Concepción/Serie Monografías, Chile.

Barriga, V.R. (2004) "La interculturalidad en tres preguntas", en Educación indígena entorno a la interculturalidad, UAM-Xochimilco/Petra Ediciones, México. 
Bonfil Batalla, Guillermo (1990) México Profundo. Una civilización negada. Ed. Grijalbo, México. Casillas Muñoz María de Lourdes y Laura Santini Villar (2009) Universidad Intercultural. Modelo Educativo, 2a . ed., coordinación General de Educación Intercultural y Bilingüe, México.

Díaz-Polanco, Héctor (1987) La teoría indigenista y la integración, Indigenismo, modernización y marginalidad. Una revisión crítica, $4^{\mathrm{a}}$ ed., Juan Pablo Editor, México.

Dietz, Gunther y Laura Selene Mateos Cortés (2011) Interculturalidad y educación intercultural en México. Un análisis de los discursos nacionales e internacionales en su impacto en los modelos educativos mexicanos, SEP.

Hernández-Loeza, Sergio Enrique (2011) La participación en los procesos de desarrollo. El caso de cuatro organizaciones de la sociedad civil en el Municipio de Cuetzalán, Puebla, Economía, Sociedad y Territorio, vol. XI, núm. 35, 95-120:

Hinojosa Rodríguez, Alejandra; Ruíz Ledesma, Javier (2015) Sustentabilidad y cosmovisión del pueblo Nahua de Cuetzalán del Progreso Puebla, Revista de Ciencia Agrícolas, vol. 1, 225230: 2007-0934.

Landázuri Benítez, Gisela (2002) Encuentros y desencuentros en Cuentepec, Morelos, UAM-X/ UAEM, México.

Quilaqueo Rapimán, Daniel (2007) "Saberes y conocimientos indígenas en la formación de profesores de educación intercultural", en Educar Em Revista, no. 29, Curtivia, recuperado de www.scielo.br/scielophp.2script=scia_arttext\&pid=S0104 (28 de mayo del 2015).

Rico P E, Santos M, Viaña M (2004) Proceso de enseñanza aprendizaje desarrollador, Editorial Pueblo y Educación. La Habana, Cuba.

Rivera N. (2003) "Fundamentos metodológicos del proceso docente-educativo. El modelo de la actividad", en: Lecturas seleccionadas material de estudio de la Maestría de Educación Médica, La Habana, Cuba.

Scott, James C. (2000) Los dominados y el arte de la resistencia, Ed. Era, México. 